\title{
Inter-centre comparison of pulsed-field gel electrophoresis for the typing of methicillin- resistant Staphylococcus aureus
}

\author{
B. D. COOKSON, P. APARICIO, ARIANE DEPLANO*, MARC STRUELENS*, R. GOERING $\dagger$ and \\ R. MARPLES
}

Laboratory of Hospital Infection, Central Public Health Laboratory, 61 Colindale Avenue, London NW9 5HT, * Unite d'Epidemiologie, Laboratoire de Microbiologie, Hospital Erasme, Brussels, Belgium and †Department of Microbiology, Creighton University, Omaha, USA

\begin{abstract}
The results of pulsed-field gel electrophoresis (PFGE) of chromosomal DNA of the same 12 methicillin-resistant $S$. aureus (MRSA) strains of diverse geographical origin, performed in three different laboratories were compared; one laboratory used fieldinversion gel electrophoresis (FIGE), one used contour clamped homogeneous electrophoresis (CHEF) and one used both (all manufactured by BioRad Laboratories Inc., Hercules, CA, USA). No single method produced the maximum number of chromosomal fragments from all isolates. In only four instances were the same number of fragments identified by any two techniques. Although there were similar trends in strain identification the results showed many discrepancies even with a three-band difference rule to discriminate between strains. Plasmids in seven of the isolates produced a fragment, but this did not affect discrimination of the study isolates. There is a great need to standardise methodology and produce a standard set of strains to assist in this process.
\end{abstract}

\section{Introduction}

Infection and colonisation of patients and staff with methicillin-resistant Staphylococcus aureus (MRSA) continues to be a problem in many parts of the world $[1,2]$. Many typing systems have been used to explore the epidemiology of MRSA and often have assisted in the interpretation of the success or otherwise of control measures. Phage typing proved valuable in this respect for many strains, particularly in the earlier period in MRSA evolution; the system is well standardised and phages are distributed to WHO centres throughout the world. However, it is not available in certain countries, and various other or additional approaches have to be used for those MRSA that type poorly at RTD or even at RTD100 [3-5].

The international transfer of MRSA is well described $[1,2,6]$. Recent changes in Eastern Europe will facilitate this spread. This will necessitate the standardisation of typing systems [7] so that crossborder spread can be monitored, the epidemiology of MRSA can be established and the information used to devise strategies for the control of the organism.

Received 31 Jan. 1995; revised version accepted 6 Aug. 1995.

Corresponding author: B. D. Cookson.
In recent years, there has been much interest in chromosomal genotyping of MRSA. The method is attractive, in that it has the potential to type all organisms. However, difficulties in interpretation of the results of digestion with restriction endonucleases that cut at frequent sites have led to the examination of alternative approaches. One of these has been to prepare chromosomal DNA in agarose blocks, which minimises shearing, and then to digest it with restriction endonucleases that cut the DNA at infrequent sites (rare cutters). The large fragments that result are 'pulsed' electrically into the agarose [8]. There are many different electrical systems that can perform this process (pulsed-field gel electrophoresis; PFGE). In field-inversion gel electrophoresis (FIGE) the electrical fields alternate forward and backward; the forward migration is achieved by the forward pulse times exceeding those in reverse. To improve the resolution of bands, pulse time duration is usually increased gradually during the run, the so-called switch time ramping. In contour clamped homogeneous electrophoresis (CHEF), hexagonally arranged electrodes and appropriate voltages are used to generate homogeneous electric fields. An angle of re-orientation of $120^{\circ}$ is created with gradations of electro-potential radiating from the positive to the negative electrodes. Macro-restriction patterns produced by this approach are usually analysed subjec- 
Table 1. MRSA isolates examined

\begin{tabular}{|c|c|c|}
\hline Isolate no. and identity & Source & Phage type* \\
\hline 1. NCTC 10442 & UK & $47 / 53 / 54 / 75 / 77 / 84 / 85$ \\
\hline 2. E2 & UK & $80 / 85 / 90 / 932$ \\
\hline 3. E03/M307 & UK & $75 / 83 \mathrm{~A} / 932$ \\
\hline 4. E12 & UK & $75 / 83 \mathrm{~A} / 85 / 83 \mathrm{C} / 932$ \\
\hline 5. $84 / 5528$ & UK & $\underline{85 / 88 \mathrm{~A} / 932 / 616 / 618 / 622 / 630 / 633}$ \\
\hline 6. $84 / 9580$ & South Africa & $\mathrm{NT}$ \\
\hline 7. $85 / 1774$ & Italy & NT \\
\hline 8. $85 / 1836$ & Germany & NT \\
\hline 9. $85 / 2147$ & Hong Kong & NT \\
\hline 10. $85 / 3619$ & Austria & NT \\
\hline 11. $85 / 4670$ & Canada & NT \\
\hline 12. $85 / 5484$ & Saudi Arabia & $85 / 88 \mathrm{~A} / 932 / 616 / 618 / 622 / 630 / 633$ \\
\hline
\end{tabular}

${ }^{*}$ Typing results are at RTD, if non-typable (NT) and typed at RT100, results are underlined.

tively. Comparison of isolates run on different gels even in the same laboratory is a distant prospect. Objective methods of measuring loci do exist but they are in their infancy and an agreed standard has not been developed. Because of the subjectivity of PFGE and the unproven ability of laboratories to produce comparable results, many refer to the technique as 'fingerprinting' although 'tentative typing' would be preferable.

It is desirable that techniques of PFGE are standardised so that typing results of MRSA isolates (and indeed other organisms) examined in different laboratories can be compared. There are several variables which must be considered in this process. These include the methods of preparing the chromosomal DNA, the source or type of restriction endonuclease, agarose and other materials used, and the pulsing conditions and equipment, which have varied in previous MRSA PFGE studies [9-11]. It would be preferable for all centres to use exactly the same materials and equipment. However, this is unrealistic; for example, equipment for FIGE is much cheaper than that for CHEF. In addition, there is the ease, cost and speed of servicing and access to replacement parts, which may vary between or even within different countries. Personal preference and previous experience must also be considered. Moreover, the level of technical expertise, training and experience may differ greatly. A number of different pulsing protocols may be required, depending on the MRSA encountered, which can vary within or between different countries [2].

This study compares the results of PFGE typing for the same organisms examined in different typing centres. The same 12 MRSA isolates were examined by three different centres with two different DNA preparation protocols and two popular approaches to PFGE (FIGE and CHEF). The isolates were chosen to represent well characterised epidemic strains and others that were geographically disparate and nontypable by phage.

\section{Materials and methods}

\section{Bacterial isolates}

To simplify the assessment, 12 MRSA isolates were selected, so that they could be run on the same gel to avoid difficulties of inter-run comparison. The criteria for the choice of the isolates were: two MRSA isolates were to be very similar, based on phage and plasmid type and antibiotic susceptibility patterns, and the others would vary in geographical origin or phage typing pattern, or both (Table 1). One investigator selected the 12 MRSA isolates; all other investigators were blinded to their identities and possible relationships. The isolates were from freeze-dried stock cultures, checked for purity and phage and antibiotic susceptibility patterns and re-freeze-dried before they were issued to the investigators.

\section{Typing methods}

All three centres had extensive experience with the techniques they employed in this study. The three centres were asked to use the same restriction enzyme

Table 2. Number of chromosome fragments detected

\begin{tabular}{lcccc}
\hline & \multicolumn{4}{c}{$\begin{array}{c}\text { Number of fragments found by technique } \\
\text { no. (technique: centre) }\end{array}$} \\
\cline { 2 - 5 } Isolate & 1 & 2 & 3 & 4 \\
no. & (FIGE:CU) & (FIGE:LHI) & (CHEF:HE) & (CHEF:LHI) \\
\hline 1 & $21^{*}$ & 13 & $16 \dagger$ & 15 \\
2 & $20^{*}$ & 13 & $17 \dagger$ & 15 \\
3 & $20^{*}$ & 11 & $15 \dagger$ & 13 \\
4 & $17^{*}$ & 13 & 15 & $16 \dagger$ \\
5 & 17 & 16 & $19^{*}$ & 16 \\
6 & $20^{*}$ & 12 & $16 \dagger$ & 14 \\
7 & $21^{*}$ & 14 & $18 \dagger$ & 14 \\
8 & 17 & 15 & $18^{*}$ & 16 \\
9 & $18^{*}$ & 14 & $17^{\dagger}$ & 16 \\
10 & $19^{*}$ & 15 & $19^{*}$ & 16 \\
11 & $19^{*}$ & 13 & $16 \dagger$ & 15 \\
12 & $19^{*}$ & $16 \dagger$ & 15 & $16 \dagger$ \\
Total & 228 & 165 & 201 & 182 \\
\hline
\end{tabular}

${ }^{*}$ Maximum number of fragments for that isolate. †The maximum number of fragments for a technique that used one run $(2,3$ or 4$)$. 
Table 3. Typing results produced by each technique

\begin{tabular}{lccc}
\hline & \multicolumn{3}{c}{$\begin{array}{c}\text { Number of strains identified by the } \\
\text { number of band differences }\end{array}$} \\
\cline { 2 - 4 } Technique no: method:centre & 1 & 2 & 3 \\
\hline 1: FIGE:CU & 12 & 12 & 10 \\
Indistinguishable isolates & & $9 \& 12$ \\
2: FIGE:LHI & 10 & $8^{*}$ & $10 \& 5$ \\
Indistinguishable isolates & $8 \& 12$ & $5 \& 12$ & $8 \& 9$ \\
& $9 \& 11$ & $8 \& 11$ & $11 \& 12$ \\
3: CHEF:HE & $9 \& 12$ & $5 \& 8$ \\
4: CHEF:LHI & 12 & 12 & 12 \\
Indistinguishable isolates & 12 & 11 & 10 \\
\hline
\end{tabular}

${ }^{*}$ Hybrid strain type present.

(Sma I) and to run the isolates (or subsets of them) as many times as they wished until they were satisfied with the results. Tables 2 and 3 also list the abbreviated centre names and techniques used, as described below.

Technique 1 (FIGE) was used by Creighton University (CU). Chromosomal DNA was prepared by the method of Goering and Winters [12] and pulsed with a FIGE Mapper (BioRad Laboratories Inc., Hercules, CA, USA) producing $16 \mathrm{~V} / \mathrm{cm}$ in $10-\mathrm{cm}$ long gels with two rapid pulsing protocols. One protocol was optimised for high $(>50 \mathrm{~kb}) \mathrm{mol}$.wt bands (initial $1.2 \mathrm{~s}$ and $0.4 \mathrm{~s}$ forward and reverse pulses linearly increased over $3 \mathrm{~h}$ to $12 \mathrm{~s}$ and $4 \mathrm{~s}$, respectively, followed by $0.75 \mathrm{~s}$ and $0.25 \mathrm{~s}$ forward and reverse pulses, respectively, for $0.5 \mathrm{~h}$ ), and the other for low $(<50 \mathrm{~kb})$ mol.wt bands $(0.4 \mathrm{~s}$ and $0.2 \mathrm{~s}$ forward and reverse pulses, respectively, over a 3.5 -h period [12].

Technique 2 (FIGE) was used in the Laboratory of Hospital Infection (LHI). DNA was prepared by the method of Goering and Winters [12] with a minor modification in agarose (BioRad Laboratories) $0.8 \%$. A FIGE (BioRad Laboratories) pulsing machine was used; the initial and final pulse times were 3 and $15 \mathrm{~s}$, the forward:reverse ratio was $3: 1$ and the running time was $18 \mathrm{~h}$ at $170 \mathrm{~V}$.

Technique 3 (CHEF) was used in centre Hospital Erasme (HE). Chromosomal DNA was prepared as described previously [11] and pulsed in a CHEF-DRII system (BioRad Laboratories) with two consecutive ramps of $12 \mathrm{~h}$ each, the first with a pulse time increase from 1 to $5 \mathrm{~s}$, the second from 15 to $30 \mathrm{~s}$ at $200 \mathrm{~V}$.

Technique 4 (CHEF) was also used in LHI. The chromosomal DNA was prepared by the same method as in technique 2 , but pulsed in agarose $1 \%$ with a CHEF-DRII system (BioRad Laboratories). The initial and final pulse times were 5 and $35 \mathrm{~s}$, respectively, over $24 \mathrm{~h}$ at $200 \mathrm{~V}$.

\section{Analysis}

The investigators analysed their gels visually. LHI and $\mathrm{CU}$ defined a locus (position of a DNA fragment) as a difference of $>0.5 \mathrm{~mm}$ in migration distance [13]. In $\mathrm{HE}$ banding patterns were compared independently by two observers. Possible doublet fragments were identified only by $\mathrm{HE}$ and were considered as single locus points for the analysis. All three centres were asked to repeat isolate runs where they were concerned about resolution of loci or if certain isolates needed to be run next to each other to resolve adequately banding pattern differences based on their previous experience. Presence or absence of a DNA fragment at each locus for each isolate was recorded and sent to LHI for analysis by an investigator who was also blinded to the identity of the MRSA. Loci data were entered into PHP-software package (Biosys, Sweden) and analysed with the Pearson product moment correlation coefficient and UPGMA clustering. Dendrogram diagrams produced with these programs were used to explore general relationships between strains. The number of band differences between the various isolates identified were also calculated in LHI and cross-tabulated as in Preheim et al. [14].

\section{Results}

No one technique detected the maximum number of fragments in all isolates (see Table 2). Technique 1 (FIGE in $\mathrm{CU}$ ) produced the largest number of fragments in all isolates except nos. 5 and 8 . However, these fragments were the combined results from two runs, designed to resolve either low or high mol.wt bands. Neither run produced as many fragments or as much discrimination as any of the other (single run) techniques (data not shown). Technique 3 produced the largest number of fragments for isolates nos. 5 and 8 and equalled technique 1 for isolate no. 10 . It performed better than the other techniques ( 2 and 4 ), as only one run was used for 10 of the isolates. However, for the remaining two isolates, technique 4 
produced more fragments for isolate no. 4 and, together with technique 2 , for isolate no. 12 . In only four instances were the same number of fragments produced by two techniques; in three of these they were from the same centre the same reagents and method of DNA preparation were used.

Clustering of the isolates by computer (Figs 1-4) indicates that isolates showed greater similarity with techniques 1,3 and 4 ; isolates 6,7 and 3 clustered together, as did nos. 5, 12 and 10 (with no. 9 for techniques 1 and 4), and isolate 1 with 2 , but isolate 8 was distinct. However, the percentage similarities of these groupings differed greatly in many instances and were usually below an $85 \%$ cut-off. The results for method 2 differed the most, although again there were some similarities (e.g., isolates 1 and 2 clustered as did isolate 7 with 3 , and 5 with 9 and 12).

These relationships were explored further by comparing the cross-tabulations of isolate band differences. In terms of discrimination, technique 3 produced the maximum number of types and technique 2 the least,

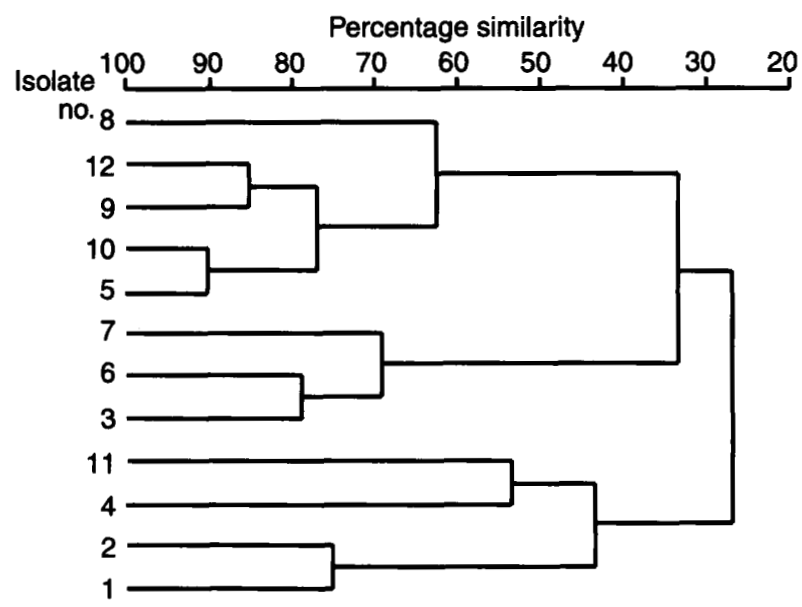

Fig. 1. Dendrogram of UPGMA clustering of results from technique 1 .

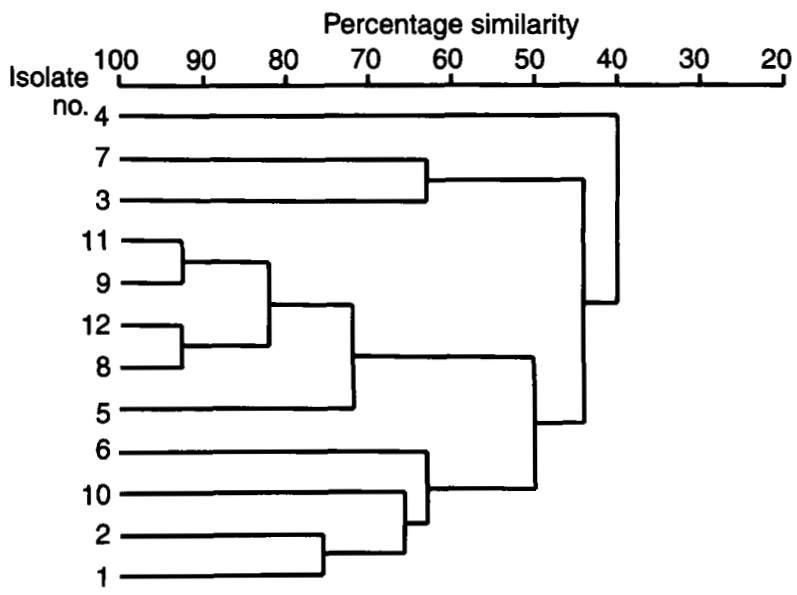

Fig. 2. Dendrogram of UPGMA clustering of - results from technique 2 .

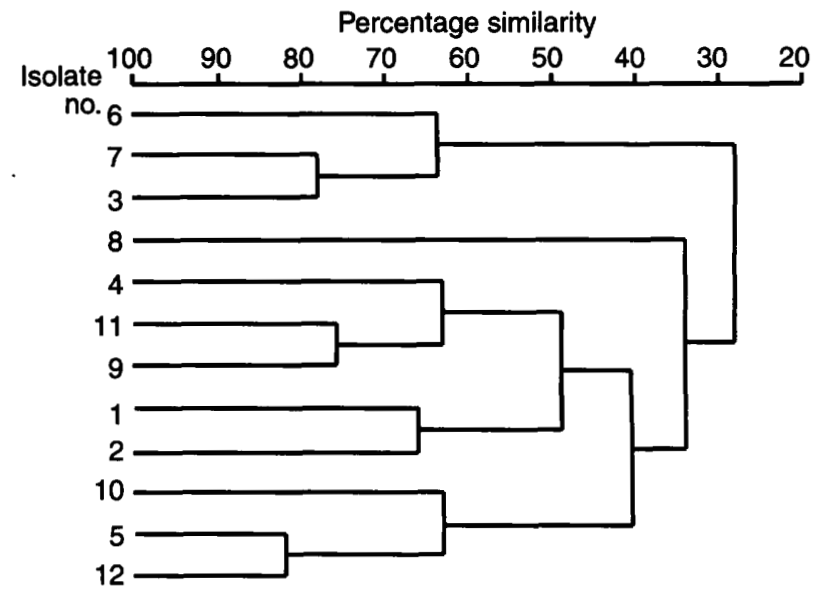

Fig. 3. Dendrogram of UPGMA clustering of results from technique 3 .

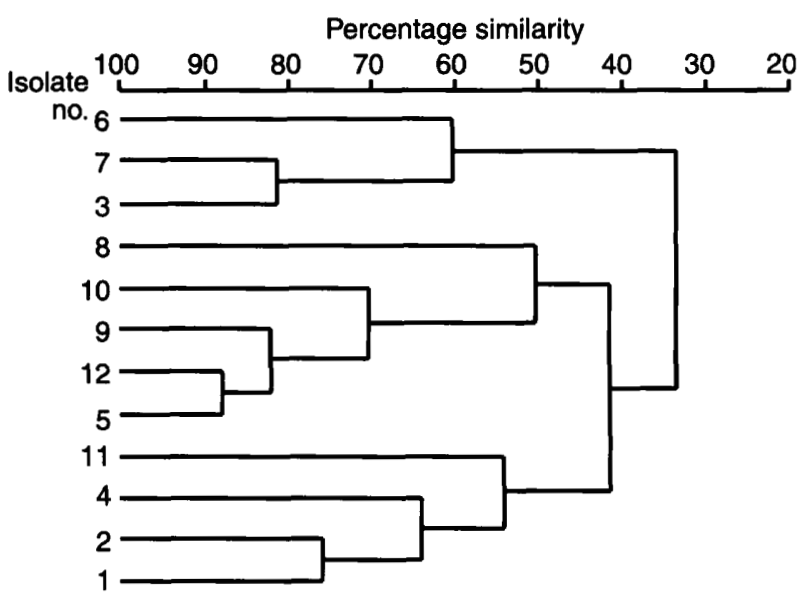

Fig. 4. Dendrogram of UPGMA clustering of results from technique 4 .

when one-, two- or three-band difference rules were employed (Table 3). The two isolates (nos 5 and 12) indistinguishable by phage susceptibility (and plasmid typing and antibiotic susceptibility patterns; data not shown), could not be distinguished by techniques 2 and 4 ; in a third (no. 3) they were the most similar (data not shown; four differences). Three techniques identified isolates 9 and 12 as similar, but in the fourth (no. 3 ) they were quite distinct (10 differences). If a greater than three band difference rule was used to define a strain type, the results became extremely difficult to interpret, in that 'hybrid' or overlapping strain types were identified; this was also evident for technique 2 with a two-band difference rule (Table 3 ). In any case, the use of such rules did not facilitate the harmonisation of typing results between the centres.

In an attempt to explore why the techniques were producing such different results, a number of possibilities were considered. A problem with the purity of the isolates seemed unlikely because they were checked and labelled very carefully before dispatch to the centres, and samples of each were re-typed before dispatch from LHI. 
To explore the possibility that plasmids were present in the isolates that might have been lost in some of the centres and thus affected the bands seen on the gels, centre CU ran plasmid preparations digested with Sma I in parallel with the chromosomal digests. Only seven of the isolates contained plasmids and all produced a single fragment of $c .40 \mathrm{~kb}$ (six of identical mol.wt). The exclusion of these loci did not alter the typing results at even the one-band difference rule level.

Co-migration of bands was the most obvious explanation for the fewer loci found in some centres. Indeed, the differences between the centres would have been larger if the duplets observed in technique 3 had not been analysed as a single band. Co-migration might have contributed to increased (subjectively assessed) density of band staining, but these differences could not be correlated with the number of different loci for each isolate in different centres. One centre (LHI), when informed of the results after completing the study, in an attempt to increase the number of loci detected to that seen in the HE CHEF patterns, decided to use a different CHEF pulsing protocol to that used in technique 4 to optimise the separation of the higher mol.wt bands. It proved impossible to predict the number of additional loci detected from band staining density observed with the initial LHI pulsing parameters. Neither was LHI able to account for all the differences seen between the two CHEF centres (LHI and HE) when these new bands were taken into account.

Finally, to explore the relative resolutions of the pulsing protocols and machines at different parts of the gel, loci greater than and less than $c$. $50 \mathrm{~kb}$ (the cut-off produced by the two FIGE runs of technique 1) were analysed. This produced even greater discrepancies in the typing results.

\section{Discussion}

The results described here underline the importance of inter-centre comparisons in the development of proposed typing techniques. PFGE and many other molecular techniques are described as 'fingerprinting' although they are perhaps more accurately described as 'tentative typing' tools. They are not yet able to compare isolates run on different gels. This study demonstrated the difficulties in comparing a small set of geographically diverse isolates that could be run on the same gel in different centres with much experience in analysis of MRSA by PFGE even with the same type of equipment.

More work will be required to explore the variation in the different techniques used in this study. The possibility that some of these bands are artifacts must be explored. To that end, exchange of DNA prepara- tions between the different centres would be valuable. The range of loci seen in this study agree with those seen in other studies $[9,15]$ with Sma I. Centres with different types of machines might use these standard strains (or DNA preparations) to develop their pulsing methods to establish the same (maximum) number of loci for each isolate. Certain other aspects of the equipment itself may have to be explored (e.g., deterioration of the electrodes with time).

Another variable that needs to be examined is the method of analysing pattern matching between isolates. Objective methods of analysing gels and other recommendations for standardising analysis [16] may facilitate this process. However, it remains to be seen what sort of discrimination will be achieved, and LHI and others [17] find much subjective interpretation is still required with these computerised methods.

This study did not attempt to compare results from different gels; the present results would indicate this to be a more distant goal.

Previous workers have found different degrees of discrimination with PFGE. Carles-Nurit and co-workers [15] compared the discrimination by PFGE of their MRSA with that of other workers [9]. However, in neither of these papers were criteria for distinguishing strains described, which in any case were different in terms of their countries of origin; it is known that MRSA strains from around the world differ in many of their characteristics [2].

This study raises the possibility that there may also be methodological variables to consider when one assesses the differing views on the applicability of PFGE to MRSA typing in addition to the confounding factors inherent in the examination of different isolates. Since this study was performed, Tenover and co-workers have compared a number of typing techniques for MRSA, including FIGE and CHEF, each performed in different centres [18]. Similar criteria for distinguishing strains were used in the two PFGE centres in that study, as was the same restriction endonuclease. However, differences were seen between the two techniques; FIGE misclassified fewer non-related strains. FIGE was thought to have the advantage in resolving lower mol.wt fragments; this did not seem to be important in the present study.

Until genotypic methods can be standardised it will be difficult to agree the criteria for defining different strains. Clearly, each point mutation in a strain could result in up to three band differences, and differences may be detectable following DNA inversion or the deletion or insertion of phage, plasmids or transposons. Lina and co-workers [19] lysogenised isolates of $S$. epidermidis and produced only a minor $50-\mathrm{kb}$ band shift in their PFGE pattern compared to the parent isolates. They further showed that this altered band 
contained phage sequences. Differences in ribotype pattern were not observed after lysogenisation with a number of phages or if a plasmid was inserted into the chromosome [14]. More recently, we have shown that up to three band differences can be produced by lysogenising an epidemic MRSA strain with phage from another variant of the same strain, re-creating the differences observed in vivo [20]. We have also shown here that plasmids may produce a band in PFGE. Currently, most investigators use one-to-three or even greater band difference rules to distinguish isolates of various species although, in two studies, up to six $D r a \mathrm{I}$ or $X b a \mathrm{I}$ band differences were seen between 'related isolates' of Pseudomonas aeruginosa from the sputum of individual patients or siblings with cystic fibrosis [21, 22]. Another approach that has been used to describe PFGE results has been to report $85 \%$ similarity of clonal groups [23]. However, this approach did not provide a solution which resolved the differences seen between the different centres in the present study.

It is now planned to extend these studies as outlined above, and assess other molecular techniques such as Western blotting and the various PCR typing methods for MRSA [23] and other organisms. Centres wishing to collaborate are requested to write to the corresponding author (B.D.C.).

\section{References}

1. Report of a combined working party of the Hospital Infection Society and British Society for Antimicrobial Chemotherapy. Revised guidelines for the control of epidemic methicillinresistant Staphylococcus aureus. J Hosp Infect 1990; 16: 351377.

2. Cookson BD, Phillips I. Epidemic methicillin-resistant Staphylococcus aureus. J Antimicrob Chemother 1990; 21 Suppl C: 57-65.

3. Cookson BD, Talsania H, Naidoo J, Phillips I. Strategies for typing and properties of epidemic methicillin-resistant Staphylococcus aureus. Eur J Clin Microbiol 1986; 5: 702-709.

4. Richardson JF, Chittasobhon N, Marples RR. Supplementary phages for the investigation of strains of methicillin-resistant Staphylococcus aureus. J Med Microbiol 1988; 25: 67-74.

5. Gaston MA, Duff PS, Naidoo J et al. Evaluation of electrophoretic methods for typing methicillin-resistant Staphylococcus aureus. J Med Microbiol 1988; 26: 189-197.

6. Townsend DE, Ashdown N, Bolton $\mathrm{S}$ et al. The international spread of methicillin-resistant Staphylococcus aureus. J Hosp Infect 1987; 9: 60-71.

7. Vaughan P, Normand C. Europe without frontiers: implications for health. London School of Hygiene and Tropical Medicine 2nd Annual Health Forum. Chichester, Wiley, 1993.
8. Schwartz DC, Cantor CR. Separation of yeast chromosome sized DNAs by pulsed field gradient gel electrophoresis. Cell 1984; 37: 67-75.

9. Ichiyama S, Ohta M, Shimokata K, Kato N, Takeuchi J Genomic DNA fingerprinting by pulsed-field gel electrophoresis as an epidemiological marker for study of nosocomial infections caused by methicillin-resistant Staphylococcus aureus. J Clin Microbiol 1991; 29: 2690-2695.

10. Prevost G, Pottecher B, Dahlet M, Bientz M, Mantz JM, Piemont Y. Pulsed field gel electrophoresis as a new epidemiological tool for monitoring methicillin-resistant Staphylococcus aureus in an intensive care unit. $J$ Hosp Infect 1991; 17: 255-269.

11. Struelens MJ, Deplano A, Godard C, Maes N, Serruys E. Epidemiologic typing and delineation of genetic relatedness of methicillin-resistant Staphylococcus aureus by macrorestriction analysis of genomic DNA by using pulsed-field gel electrophoresis. J Clin Microbiol 1992; 30: 2599-2605.

12. Goering RV, Winters MA. Rapid method for epidemiological evaluation of gram-positive cocci by field inversion gel electrophoresis. J Clin Microbiol 1992; 30: 577-580.

13. Cookson BD, Stapleton P, Ludlam H. Ribotyping of coagulasenegative staphylococci. $J$ Med Microbiol 1992; 36: $414-419$.

14. Preheim L, Pitcher D, Owen R, Cookson B. Typing of methicillin resistant and susceptible Staphylococcus aureus strains by ribosomal RNA gene restriction patterns using a biotinylated probe. Eur J Clin Microbiol Infect Dis 1991; 10: $428-436$.

15. Carles-Nurit MJ, Christophle B, Broche S, Gouby A, Bouziges $\mathrm{N}$, Ramuz M. DNA polymorphisms in methicillin-susceptible and methicillin-resistant strains of Staphylococcus aureus. J Clin Microbiol 1992; 30: 2092-2096.

16. van Embden JDA, Cave MD, Crawford JT et al. Strain identification of Mycobacterium tuberculosis by DNA fingerprinting: recommendations for a standardized methodology. $J$ Clin Microbiol 1993; 31: 406-409.

17. Dijkshoorn L, James PA, Maquelin K, Tew LE. An endemic episode of resistant Acinetobacter in a district general hospital investigated by pattern analysis of protein profiles and antibiograms. Programme and Abstracts of the Third International Meeting on Bacterial Epidemiological Markers, Cambridge, 1994. Abstract PA52:101.

18. Tenover FC, Arbeit R, Archer G et al. Comparison of traditional and molecular methods of typing isolates of Staphylococcus aureus. J Clin Microbiol 1994; 32: 407-415.

19. Lina B, Bes M, Vandenesch F, Greenland T, Etienne J, Fleurette J. Role of bacteriophages in genomic variability of related coagulase-negative staphylococci. FEMS Microbiol Lett 1993; 109: 273-278.

20. Reith S, Marples RR, Cookson BD. The epidemiology and evolution in typing characteristics of an epidemic methicillinresistant Staphylococcus aureus (EMRSA-16). Programme and Abstracts of the Third International Meeting on Bacterial Epidemiological Markers, Cambridge, 1994. Abstract PA32:91.

21. Grothues D, Koopmann U, von der Hardt H, Tümmler B. Genome fingerprinting of Pseudomonas aeruginosa indicates colonization of cystic fibrosis siblings with closely related strains. J Clin Microbiol 1988; 26: 1973-1977.

22. Struelens MJ, Schwam V, Deplano A, Baran D. Genome macrorestriction analysis of diversity and variability of Pseudomonas aeruginosa strains infecting cystic fibrosis patients. J Clin Microbiol 1993; 31: 2320-2326

23. Struelens MJ, Bax R, Deplano A, Quint WGV, van Belkum A Concordant clonal delineation of methicillin-resistant Staphylococcus aureus by macrorestriction analysis and polymerase chain reaction genome fingerprinting. J Clin Microbiol 1993, 31: $1964-1970$. 